\title{
Exciton localization and interface roughness in growth-interrupted GaAs/AIAs quantum wells
}

\author{
Leosson, Kristjan; Jensen, Jacob Riis; Langbein, Wolfgang Werner; Hvam, Jørn Märcher
}

Published in:

Physical Review B Condensed Matter

Link to article, DOI:

10.1103/PhysRevB.61.10322

Publication date:

2000

Document Version

Publisher's PDF, also known as Version of record

Link back to DTU Orbit

Citation (APA):

Leosson, K., Jensen, J. R., Langbein, W. W., \& Hvam, J. M. (2000). Exciton localization and interface roughness in growth-interrupted GaAs/AIAs quantum wells. Physical Review B Condensed Matter, 61(15), 10322-10329. https://doi.org/10.1103/PhysRevB.61.10322

\section{General rights}

Copyright and moral rights for the publications made accessible in the public portal are retained by the authors and/or other copyright owners and it is a condition of accessing publications that users recognise and abide by the legal requirements associated with these rights.

- Users may download and print one copy of any publication from the public portal for the purpose of private study or research.

- You may not further distribute the material or use it for any profit-making activity or commercial gain

- You may freely distribute the URL identifying the publication in the public portal 


\title{
Exciton localization and interface roughness in growth-interrupted GaAs/AlAs quantum wells
}

\author{
K. Leosson, J. R. Jensen, W. Langbein,* and J. M. Hvam \\ Research Center COM, Technical University of Denmark, Building 349, DK-2800 Lyngby, Denmark
}

(Received 19 July 1999; revised manuscript received 19 November 1999)

\begin{abstract}
We have used photoluminescence spectroscopy to investigate the influence of interface roughness in GaAs/ AlAs quantum wells on their optical properties over a wide continuous range of well thicknesses. In order to compare different correlation lengths of the in-plane disorder potential, the wells were fabricated with growth interruption at both, one, or neither of the interfaces. Growth-interruption increases the correlation length of the monolayer-island structure on the surface, which gives rise to a long-range interface roughness after overgrowth. The relation between the correlation lengths of the in-plane disorder potential and the exciton localization length determines the spectral shape of the exciton luminescence. When the correlation length of the in-plane disorder potential is larger than the exciton localization length, the excitonic spectrum splits up into discrete peaks, stemming from regions differing in effective thickness by an integral number of monolayers. The energies of monolayers peaks, taking into account the in-plane localization energy, are found to be reproducible in wafers grown under similar conditions. We conclude that atomically smooth growth islands are formed on both AlAs and GaAs surfaces after growth interruption. During overgrowth, surface segregation leads to the generation of an atomic-scale disorder in the first overgrown monolayers. This results in an additional in-plane disorder potential with a much shorter correlation length than the original surface. It also modifies the shape of the well potential in the growth direction, as we have modelled by growth simulations, blueshifting the excitonic transition energies with respect to a square-well model.
\end{abstract}

\section{INTRODUCTION}

Interface roughness is an important parameter for the optical and electrical properties of quantum wells and, consequently, for quantum-well based devices. The observed photoluminescence (PL) spectrum of quantum wells with imperfect interfaces is largely determined by how the length scales of the interface roughness compare to the diameter, localization length and diffusion length of the quantum-well excitons. When quantum wells are grown with molecularbeam epitaxy (MBE), the interface roughness can be tailored to some extent by interrupting the growth at the heterointerfaces, allowing for a restructuring of the free surface, mainly by surface diffusion. It was realized early on that growth islands with sizes comparable to or larger than the exciton diameter can be created in this way, resulting in a splitting of the PL into several lines of reduced inhomogeneous linewidth. ${ }^{1}$ Initially, such narrow luminescence lines were assigned to laterally extended quantum well regions with a well-defined monolayer (ML) thickness. ${ }^{1,2}$

More detailed investigations on these "monolayer peaks" in growth-interrupted (GI) quantum wells revealed, however, that narrow exciton luminescence was not necessarily indicative of quantum wells having perfect interfaces and integer monolayer widths. Gammon et al. ${ }^{3}$ demonstrated a wafer-towafer variation in absolute energies of ML peaks in GI GaAs/AlAs wells grown under identical conditions. Warwick et ll $^{4}{ }^{4}$ observed a significant variation in peak energy in $\mathrm{Al}_{0.37} \mathrm{Ga}_{0.63} \mathrm{As} / \mathrm{GaAs}$ wells in a single sample, which could not be explained by alloy fluctuations. The simplest model which can account for this behavior is that of bimodal interface roughness. ${ }^{4}$ It is now commonly accepted that discrete luminescence lines can originate from extended quantum well regions that differ in effective thickness by approxi- mately one monolayer and exhibit nanoroughness on a length scale smaller than the exciton diameter. Optically, the presence of nanoroughness has been inferred from measurements of GI GaAs quantum wells with $\mathrm{Al}_{x} \mathrm{Ga}_{1-x} \mathrm{As}$ $(x \leqslant 0.4)$ barriers $^{5-10}$ as well as pure AlAs barriers. ${ }^{11,12}$

Scanning tunneling microscopy studies indicate that the as-grown GaAs surface has atomically-flat islands which, after growth interruption, can reach lateral sizes of tens or hundreds of nanometers, ${ }^{13}$ possibly with a distribution of ML-deep holes much smaller than the exciton diameter. ${ }^{14}$ The as-grown AlAs surface exhibits a higher degree of roughness on the nanometer scale, even after growth interruption, due to the smaller surface mobility of $\mathrm{Al}^{12}$ Nevertheless, atomically smooth growth islands as large as $15 \mathrm{~nm} \times 40 \mathrm{~nm}$ on an $\mathrm{Al}_{0.35} \mathrm{Ga}_{0.65}$ As surface have been reported. ${ }^{13}$

In order to clarify the relationship between surface roughness during growth and the final interface structure, we have used PL and microphotoluminescence ( $\mu$-PL) spectroscopy to characterize GaAs/AlAs quantum wells fabricated with growth interruption at one, both, or neither of the interfaces. The wells have a wide range of thicknesses, varying continuously between approximately 4 and $11 \mathrm{~nm}$ (for thicknesses under $4 \mathrm{~nm}$, the indirect barrier material results in the formation of type-II quantum wells ${ }^{12}$ ). The use of binary barrier and well materials eliminates the effects of alloy disorder, making the results more reproducible. By interrupting the MBE growth, we tune the correlation length of the quantum well potential through several distinct regimes of interface roughness. Using recent theoretical results ${ }^{15}$ we identify these regimes with approximate length scales. Contrary to previous work in the field, we demonstrate that when sufficiently large growth islands are formed, reproducible $\mathrm{ML}$ peak positions can be achieved over a large range of quan- 
tum well thicknesses, consistent with the formation of monolayer-flat islands on both AlAs and GaAs surfaces after GI. From growth simulations, we deduce that segregation effects during overgrowth are generating atomic-scale interface roughness, resolving the apparent contradiction between atomically smooth as-grown surfaces and the observed nanoroughness of quantum well interfaces.

\section{EXPERIMENT}

Single GaAs quantum wells, nominally 10,7 , and $5 \mathrm{~nm}$ wide, were MBE grown at $630^{\circ} \mathrm{C}$ on two-inch GaAs (100) wafers using pure AlAs barriers. One wafer (Wafer 1) was grown continuously, without growth interruption. Three wafers were grown with a 120 s GI before (Wafer 2), after (Wafer 3) and both before and after (Wafer 4) growing the wells. Short-period GaAs/AlAs superlattices (SPSL's) were grown between wells in order to trap impurities and improve the surface structure. Rotation of the substrate was stopped only during the growth of the wells in order to achieve a continuous variation in well thickness across the wafer while maintaining a constant barrier width. Growth rates were calibrated using reflectance high-energy electron diffraction (RHEED) on a reference wafer. The nominal growth rates were $0.8 \mathrm{ML} / \mathrm{s}$ and $0.3 \mathrm{ML} / \mathrm{s}$ for GaAs and AlAs, respectively, and a $30 \%$ variation in growth rate was observed across the wafer. A standard V/III flux ratio of $8-10$ was used. For comparison, a wafer with four GaAs quantum wells, growth-interrupted at both interfaces, was fabricated in a later run, under similar growth conditions (Wafer 5). The nominal quantum well thicknesses were $11,8.5,6.5$, and $5 \mathrm{~nm}$. Wafer 5 was grown with narrow $(8 \mathrm{~nm})$ AlAs barriers and $50 \mathrm{~nm} \mathrm{GaAs}$ spacers but no SPSL's between wells.

Conventional PL measurements were carried out at sample temperatures of $10-50 \mathrm{~K}$. In addition, micro-PL spectra were measured at $10 \mathrm{~K}$ on selected samples. The samples were cooled in a closed-cycle He cryostat and excited with a He-Ne laser, focused to a $50 \mu \mathrm{m}$ spot. The PL was dispersed in a spectrometer and detected with a cooled charge coupled device array. The spectral resolution of the detection system was $\approx 1 \AA(0.2 \mathrm{meV})$. In $\mu$-PL measurements, the excitation beam and PL were passed confocally through a 0.85 NA objective located inside the cryostat, giving an excitation spot diameter and a spatial resolution close to $0.5 \mu \mathrm{m}$.

\section{RESULTS}

Figure 1 shows PL spectra from the three single quantum wells measured at similar positions on Wafers 1 to 4 . The continuously grown Wafer 1 exhibits nearly Gaussian luminescence lines for all well widths. At first glance, no large difference is observed in Wafer 2, when MBE growth is interrupted only at the bottom (inverted) interface. However, as shown below, systematic variations in linewidth are observed when the PL is measured at different positions on the wafer. In the third case, where growth is interrupted only at the top (normal) interface, the PL peaks split into a doublet, most clearly seen in the $7 \mathrm{~nm}$ well. Only when growth is interrupted at both interfaces does the luminescence exhibit narrow peaks with discrete energies. Such a splitting of the

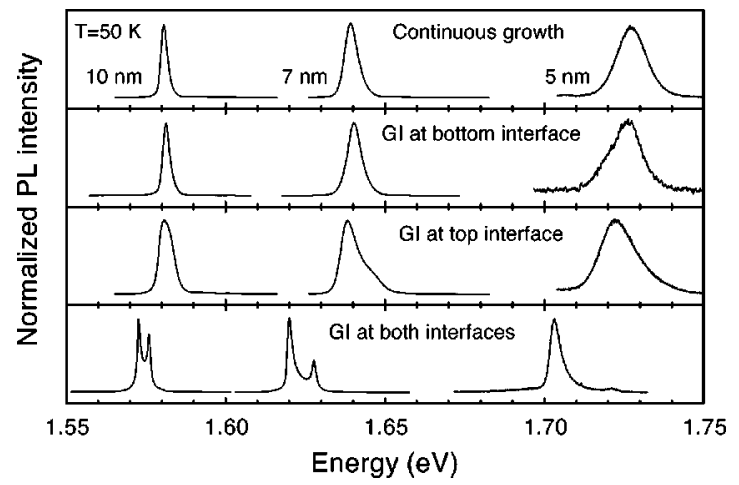

FIG. 1. PL, measured at $50 \mathrm{~K}$, from quantum wells of equal thickness but grown with 120-s growth interrupts at neither, one, or both interfaces, as indicated. The spectra from each well have been normalized with respect to the peak height.

PL from each well into two or three peaks is observed on Wafer 4 . The highest energy peak is well fitted by a Lorentzian function whereas the peaks at lower energies are asymmetric, with a tail on the high energy side. Identical results were obtained for Wafer 5 (not shown).

The variation of the PL from single quantum wells along a $5 \mathrm{~mm}$ section of each wafer is shown in Fig. 2. The spectra are taken at comparable positions on Wafers 1 to 4 . The total scan distance corresponds to a quantum well thickness

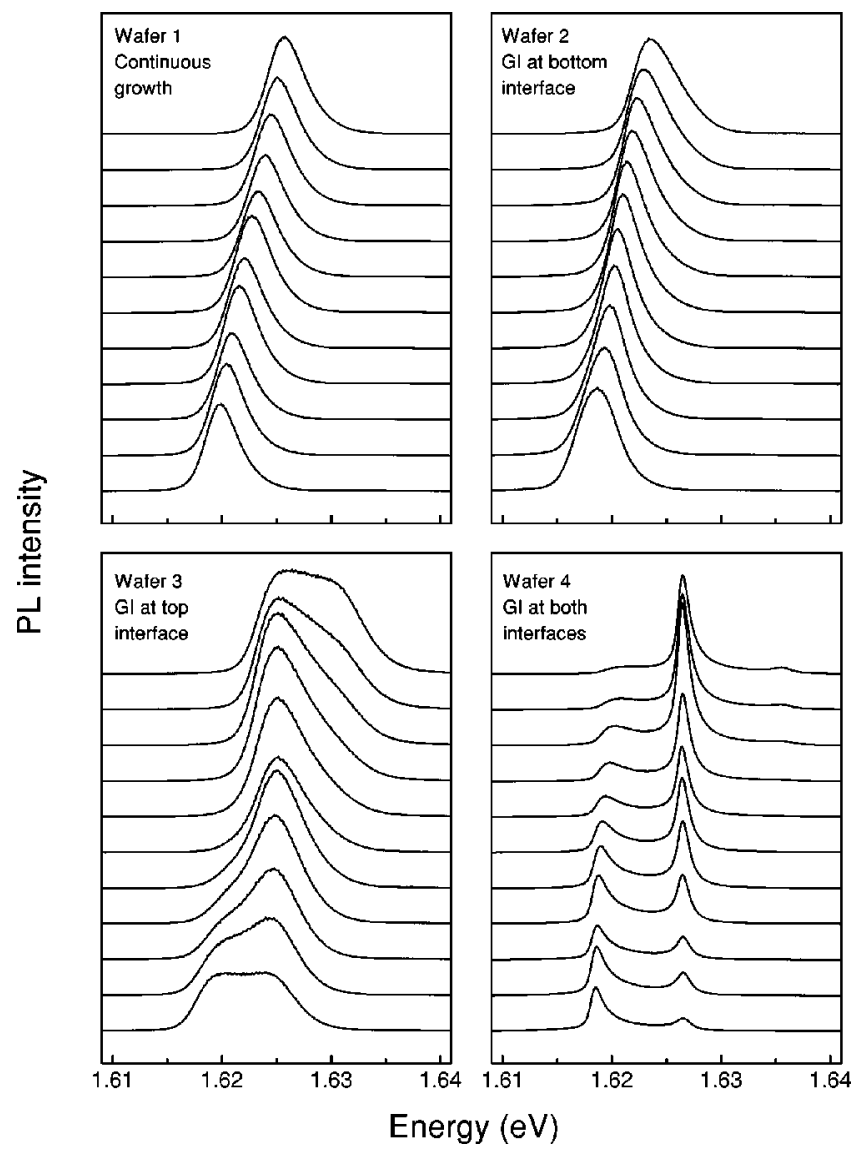

FIG. 2. Single quantum well PL spectra sampled over a $5 \mathrm{~mm}$ region on the wafers at $50 \mathrm{~K}$. The total scan distance represents a thickness change of approximately one monolayer. The average well thickness is $6 \mathrm{~nm}$. 

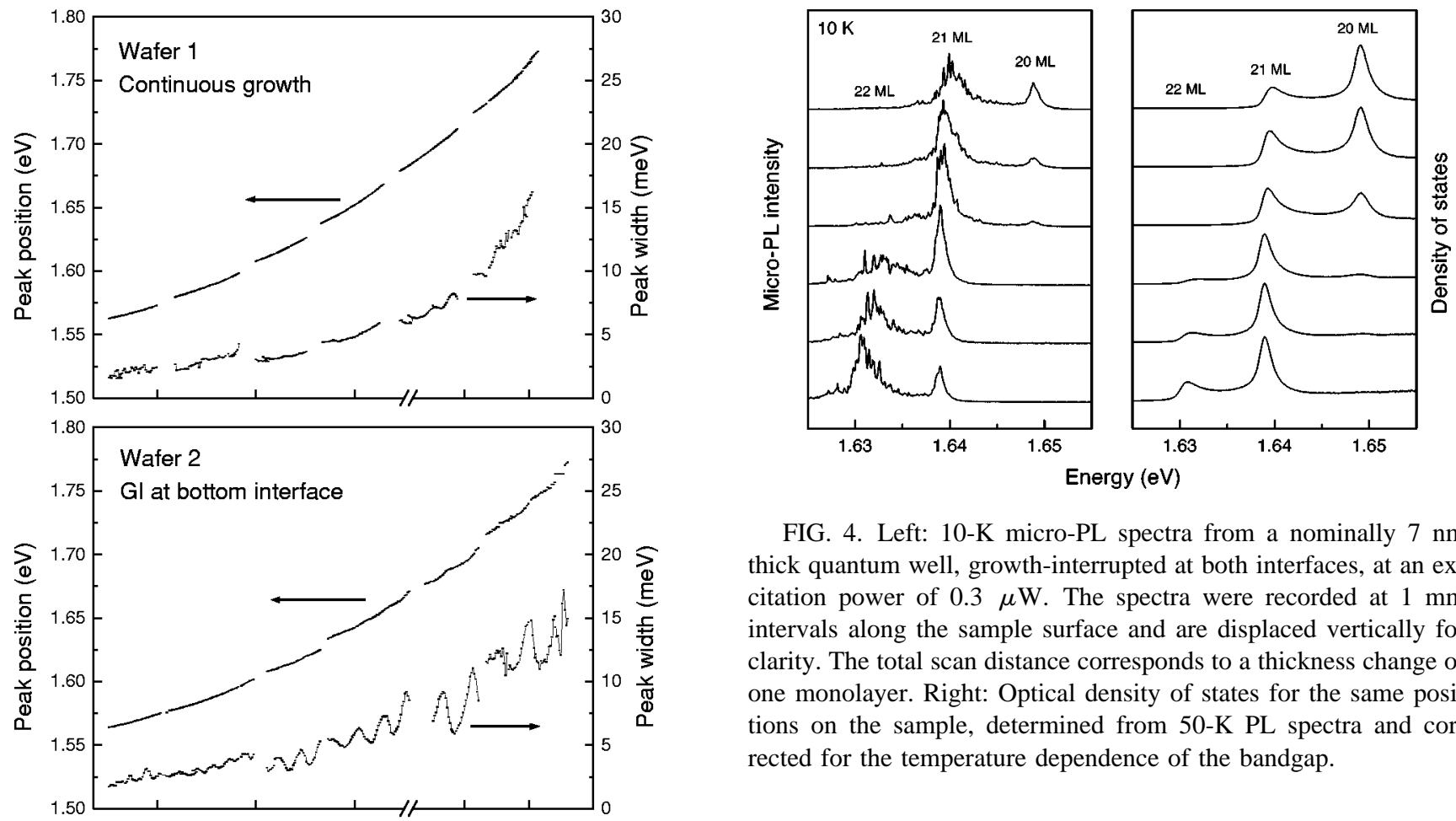

FIG. 4. Left: $10-\mathrm{K}$ micro-PL spectra from a nominally $7 \mathrm{~nm}$ thick quantum well, growth-interrupted at both interfaces, at an excitation power of $0.3 \mu \mathrm{W}$. The spectra were recorded at $1 \mathrm{~mm}$ intervals along the sample surface and are displaced vertically for clarity. The total scan distance corresponds to a thickness change of one monolayer. Right: Optical density of states for the same positions on the sample, determined from 50-K PL spectra and corrected for the temperature dependence of the bandgap.

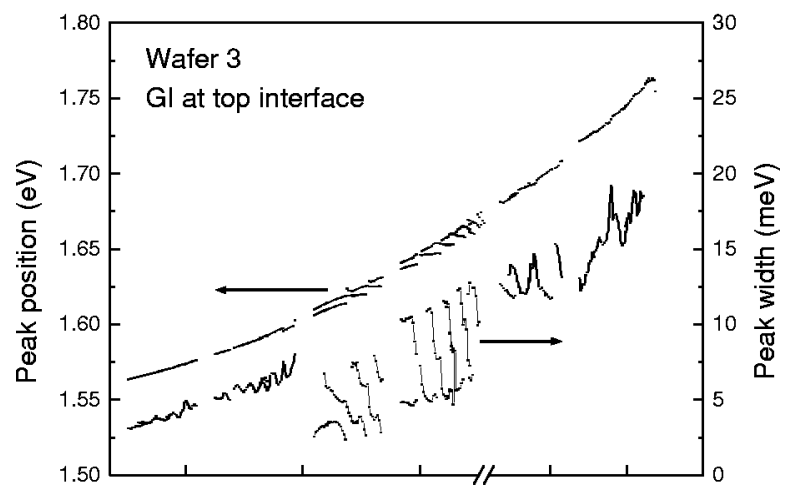

across the wafer. The position on the wafer has been converted into an approximate thickness scale. For Wafers 1 and 2 , the PL signal was fitted with a single Gaussian peak. In the case of Wafer 3, the data was fitted with two Gaussian peaks for intermediate well thicknesses $(5-8 \mathrm{~nm})$. The position and full width at half maximum (FWHM) of each peak is indicated in this thickness range. For narrower and wider wells, the peaks could not be resolved and the position of the maximum luminescence and the FWHM of the total signal are given. For Wafer 4, where growth was interrupted at both interfaces, the positions and FWHM of the individually fitted Lorentzian peaks are shown.

The left graph in Fig. 4 shows $\mu$-PL spectra measured at $10 \mathrm{~K}$ on the nominally $7 \mathrm{~nm}$-thick quantum well in Wafer 4. The PL was excited and detected confocally, with a resolution of approximately $0.5 \mu \mathrm{m}$ and the displayed spectra were recorded at $1 \mathrm{~mm}$ intervals along the sample surface. As the probe is scanned in the direction of decreasing well thickness, each ML peak in the $\mu$-PL spectra first appears as a single unresolved peak with a FWHM around $1 \mathrm{meV}$, increasing in intensity to a maximum value without shifting in energy. As the maximum intensity drops, the peak splits up into narrow lines with widths below our resolution limit. The narrow-line pattern varies with position on the sample and individual lines arise from regions with sizes below our spatial resolution. As the well thickness decreases further, the single lines spread over a larger energy range, the center of the distribution shifts to higher energies and the total intensity drops. For $\mu$-PL measurements an excitation power of $0.3 \mu \mathrm{W}$ was used. A significant modification of the narrowline spectrum due to saturation of states was observed when the excitation power exceeded $3 \mu \mathrm{W}$. Figure 4 also shows the exciton optical density of states (ODOS) for corresponding positions on the sample. The ODOS was obtained from the $50 \mathrm{~K}$ PL spectra assuming a Boltzmann distribution of carriers. The spectra were shifted in energy to compensate for the change in the GaAs bandgap with temperature. To 
confirm that the excitons are in thermal equilibrium at $50 \mathrm{~K}$, we performed a temperature dependent photoluminescence study of adjacent ML peaks (16 and $17 \mathrm{ML}$ ) with a relatively large splitting (around $11 \mathrm{meV}$ at the position measured). The integrated intensity ratio of the two peaks essentially follows the form given by Melliti et al. ${ }^{16}$ with a linear temperature dependence above $30 \mathrm{~K}$ characterized by an activation energy of around $8 \mathrm{meV}$.

Our main observations from the PL spectra can be summarized as follows:

(i) The continuously grown single quantum wells exhibit a smooth increase in transition energy and linewidth with decreasing well thickness. The inhomogeneous linewidth broadening corresponds to an exciton-averaged well thickness variation of less than a monolayer. Anomalously increased linewidth, along with a drop in luminescence efficiency, is observed close to the edges of the wafer. The line shape is discussed in more detail in the following section.

(ii) Growth interruption at the bottom interface introduces only a slight modulation of the peak position as a function of well width. The peak width, however, oscillates strongly with the well thickness, in some cases dropping below the corresponding linewidth of the continuously grown well. A reduction in PL excitation (PLE) linewidth in similarly prepared samples reported by Zhang et al. ${ }^{17}$ is consistent with this observation.

(iii) In the case of growth interruption at the top interface, the PL is split into a doublet. The average PL energy increases continuously with a weak modulation, similar to the previously described case. However, when two peaks are resolved, each peak shifts to higher energy as it gains intensity and then stays pinned or moves to lower energies as the intensity drops again, with a decrease in peak width. Even more pronounced "sawtooth" behavior of this kind was reported by Gammon et al. in similar quantum wells and attributed to gradients in barrier thickness. ${ }^{3}$ Our observations show, however, that the effect persists with AlAs layers of constant thickness. Furthermore, we note that in the ODOS calculated from the PL spectra each peak moves monotonically upwards in energy with decreasing well thickness.

(iv) When growth is interrupted at both top and bottom interfaces, two or three narrow PL lines are observed simultaneously. As the probe is scanned across the surface, the peak position is generally pinned within $\pm 0.2 \mathrm{meV}$ until the peak has lost approximately half its maximum intensity, then it broadens and shifts gradually to higher energies. The minimum linewidths stay constant at around $1.3 \mathrm{meV}$, increasing to about $5 \mathrm{meV}$ only for the narrowest wells, as also observed in Ref. 18. Similar discrete peaks were also measured in Wafer 5, with minimum linewidths around $0.8 \mathrm{meV}$. We attribute the smaller linewidth in these wells to reduced exciton-electron scattering, ${ }^{19}$ confirmed by the observation of trionic PL in Wafer 4 at low temperatures. Due to the narrow barriers, charging effects are less important in the quantum wells of Wafer 5, which do not show trionic PL at low temperatures.

(v) Micro-PL spectroscopy reveals that the broadening and shifting of ML peaks is observed simultaneous to their further splitting into sharp PL lines, arising from spatially localized exciton states. When compared with the exciton ODOS, the $\mu$-PL curves confirm the localization of the ex- citons into small islands, in which the in-plane quantization supersedes the effect of nanoroughness within the islands.

\section{ANALYSIS}

To determine the degree of interface roughness and exciton localization in our continuously grown samples we use the line shape model of Schnabel et al. ${ }^{20}$ to fit the PL peaks. This model takes into account the violation of wave-vector conservation due to partial localization of the exciton's center-of-mass wave function. For the ground state transition, the optical density for a disordered potential with a mean energy $E_{0}$ can be calculated to

$$
\bar{\alpha}(E) \propto \frac{1}{2 \eta}\left[1+\operatorname{erf}\left(\frac{E-E_{0}}{\sigma_{E}}-\frac{\sigma_{E}}{2 \eta}\right)\right] \exp \left[\left(\frac{\sigma_{E}}{2 \eta}\right)^{2}-\frac{E-E_{0}}{\eta}\right] .
$$

We estimate $\bar{\alpha}$ by calculating the ODOS from our $50-\mathrm{K}$ PL spectra, as previously described, and fit Eq. (1) to the result. The relevant fitting parameters are the standard deviation of the potential variation, $\sigma_{E}$, and a localization energy parameter, $\eta=\hbar^{2} \Delta K^{2} / 2 M$, derived from the wavevector uncertainty $\Delta K$, with $M$ being the exciton mass. For the narrowest continuously grown wells, we find a potential variation around $4 \mathrm{meV}$, decreasing to $1 \mathrm{meV}$ for the widest wells. The localization energy parameter follows a similar dependence on well width, with the ratio $\sigma_{E} / \eta$ being nearly constant for all wells and equal to $0.67 \pm 0.03$. The momentum uncertainty in the model can be converted to a minimum localization radius, $\Delta R$, via the uncertainty relation, yielding lower limits for localization radii at $3.5 \mathrm{~nm}$ for the narrowest well, increasing to $8 \mathrm{~nm}$ for the widest.

Energies of ML peaks measured in Wafers 4 and 5 are plotted in Fig. 5(a). The measured data points correspond to the energies at which the PL peaks are pinned before blueshifting as the peaks lose intensity. Since the only periodic change over the different positions is the well thickness in monolayers, we assign the neighboring peak positions to quantum wells differing in thickness by one monolayer. This assignment is in agreement with the RHEED growth calibration. From Fig. 3 it is evident that due to the peak shift over the average well thickness, a submonolayer splitting between peaks is observed when comparing the positions of the different peaks for the same average thickness, i.e., in the PL measured at one position. Such submonolayer splitting (typically $0.8-0.9$ ML) has been previously reported in literature. ${ }^{2,4,6,8,10}$ In order to determine the absolute thickness of the wells, we find the position on the wafer where the thickness difference between wells (counted in ML steps in the PL spectra) matches exactly the RHEED-calibrated thickness difference. At this position, the actual well thickness equals the nominal thickness and, therefore, we can assign absolute quantum well thicknesses to the series of ML peaks, accurate to within $1 \mathrm{ML}$.

We find an excellent agreement between ML peak positions within each wafer and between wafers. Typical variations in peak position between Wafers 4 and 5 are of the same order as the variation within the wafers (under 0.5 $\mathrm{meV}$ ). For intermediate well thicknesses, the agreement is better than $0.1 \mathrm{meV}$. For wells with thickness around $7 \mathrm{~nm}$, 


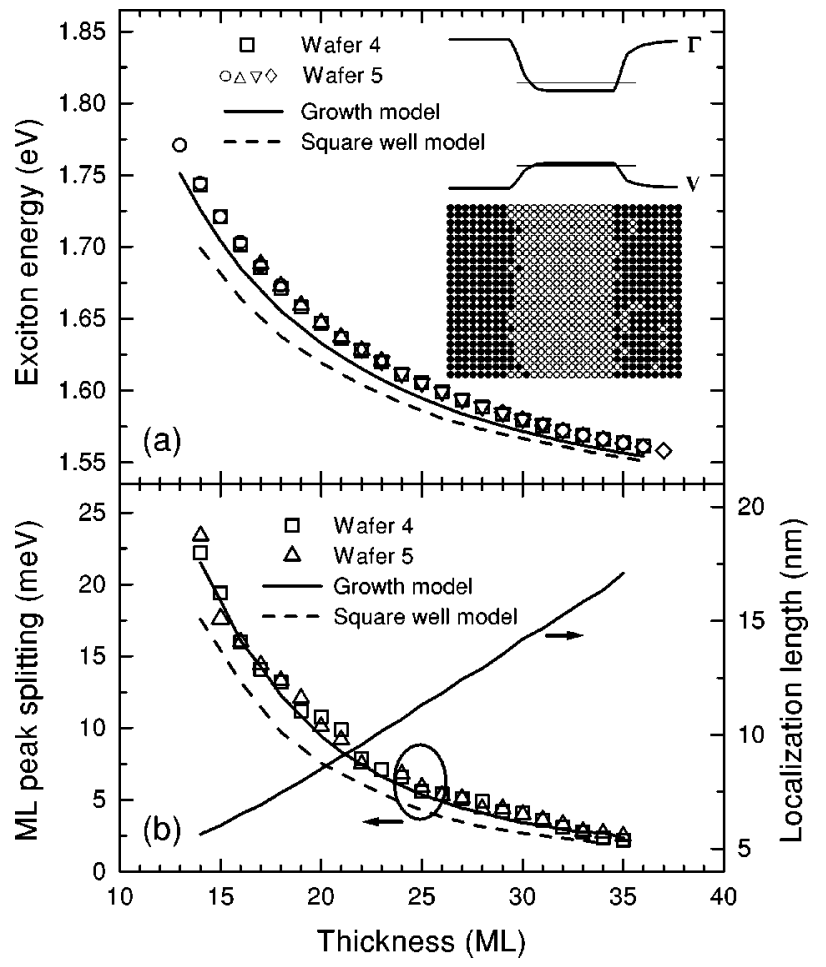

FIG. 5. (a) Discrete PL peak energies in growth-interrupted Wafer 4 (squares) and Wafer 5 (circles, diamonds, and triangles) demonstrating the reproducibility of peak positions measured in different wells and on different wafers. Also shown are calculated transition energies for a square well potential (dashed line) and a well potential where segregation effects are included (solid line). The diagram on the right schematically illustrates the calculated segregation in a $14 \mathrm{ML}$ well with initially flat growth surfaces, along with the resulting well potential. (b) Experimental and theoretical values for the monolayer peak splitting. The calculated ML splitting is in excellent agreement with the measured values when segregation is included. The exciton localization length, determined from the peak splitting, is also shown.

this corresponds to a maximum exciton-averaged thickness difference of less than $0.02 \mathrm{ML}$. It should be emphasized that several months passed between the growth of Wafers 4 and 5 and that the wafers have different barrier configurations and different nominal well thicknesses. The largest deviations (up to $0.3 \mathrm{ML}$ ) are observed for the narrowest wells $(<16 \mathrm{ML})$ and for peaks measured close to the wafer edges.

In order to calculate the transition energies of our quantum wells we used an effective-mass model with two types of well potentials: (i) a simple finite-barrier square well potential with integral ML thickness and (ii) a nonabrupt potential obtained from a surface segregation model, where the possibility of cation interchange at the interfaces during overgrowth is taken into account. The calculation of the electronic states was carried out assuming an isotropic conduction band and using a six-band $\mathbf{k} \cdot \mathbf{p}$ approximation for the valence band. The physical parameters used in the calculation, along with a description of the segregation model, are provided elsewhere. ${ }^{21}$ Well-width dependent heavy-hole exciton binding energies were calculated using the interpolation formula provided by Gurioli et $a l^{22}$ The variation of the GaAs band gap with temperature was determined from the

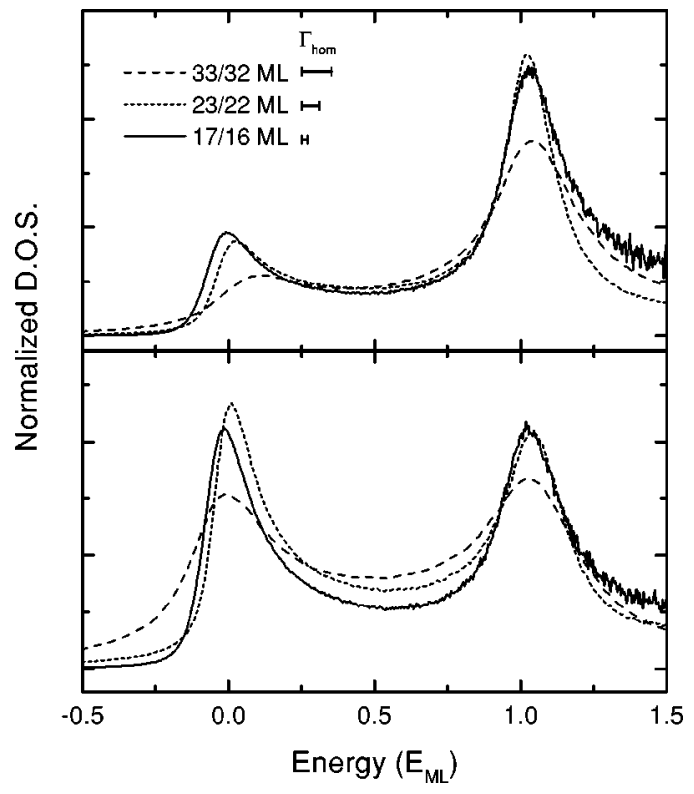

FIG. 6. Exciton optical density of states in wells of different thicknesses, plotted on a normalized energy scale. The graphs represent different island densities; $30 \%$ (top) and 50\% (bottom). The spectra are normalized with respect to the total number of states. Horizontal bars indicate an upper limit of the homogeneous linewidth on each energy scale.

shift in the near-bandgap luminescence of the GaAs substrate, giving $E_{g}(0 \mathrm{~K})-E_{g}(50 \mathrm{~K})=3.0 \mathrm{meV}$. The results of the calculations are plotted in Fig. 5(a). We observe a significant blueshift of transition energies when segregation effects are included, up to $27 \mathrm{meV}$ for $14 \mathrm{ML}$ wells. Calculated ML peak splittings for the two types of well potential and measured values from Wafers 4 and 5 are plotted as a function of well thickness in Fig. 5(b). Excellent agreement between calculated and measured values of the peak splitting is obtained when segregation effects are taken into account. In Fig. 5(a) we also show schematically the calculated interface structure and resulting well potential for a $14 \mathrm{ML}$ quantum well, assuming initially perfect growth surfaces. This clearly shows the importance of segregation, which introduces nanoroughness on the interface, even in the case of growth-interrupted QW's.

In Fig. 6, we show the ODOS for three different well widths from Wafer 4 on a monolayer energy scale. By carefully selecting the positions on the wafer, we compare spectra with average well widths of $(n+0.3)$ ML and $(n$ $+0.5)$ ML, with $n=16,22$, and 33. In the former case, the low-energy peak shifts to higher energy and becomes less defined with increasing well thickness while the position of the high-energy peak remains fixed. In the latter case, peak positions are unaffected but more states appear between the monolayer peaks. We note that this modification cannot be accounted for by the relative increase of the homogeneous linewidth $\Gamma_{\text {hom }} \leq 0.7 \mathrm{meV}$ (Ref. 23), which is indicated for reference in the figure. We attribute the observed changes in the ODOS to the larger localization length in wider wells, as discussed in the following section.

\section{DISCUSSION}

In previous literature, simple quantitative models have generally been employed in order to explain the experimen- 
tally observed ML peak splitting in the exciton luminescence from growth-interrupted quantum wells. These models assume that one or both interfaces consist of extended monolayer-high but atomically rough growth islands with sizes comparable to or larger than the exciton radius. $^{2-4,7,8,11-13}$ Sharp transition lines observed in micro-PL have furthermore been attributed to roughnessinduced localization of exciton states. ${ }^{24-27}$

The case of a realistic well potential with a disorder correlation length smaller than the exciton radius has been treated theoretically in some detail. ${ }^{28,29}$ Due to the small length scales of the disorder, these studies do not predict a splitting of the optical exciton density of states. In a recent work of Castella and Wilkins, ${ }^{15}$ however, the problem is studied for a wider range of correlation lengths. Their analysis indicates (a) that the energy distribution of exciton states is mainly dependent on the ratio of island size (correlation length) to localization length, rather than the ratio of island size to exciton radius and (b) that ML peaks appear when the island size is similar to or larger than the localization length. The localization length represents the minimum lateral extension of a disk-shaped potential fluctuation which creates a bound state in the quantum-well plane, ${ }^{15}$

$$
\xi_{0}=\frac{\pi \hbar}{\sqrt{2 M V_{0}}} .
$$

$V_{0}$ is the strength of the confining potential, which is given by the monolayer peak spacing when the island size is larger than the exciton diameter. As a result, the localization length increases considerably with well thickness and larger growth islands are required in wider wells to observe ML splitting. In Fig. 5(b) we plot $\xi_{0}$ for the quantum wells of Wafers 4 and 5. The localization length varies from $6 \mathrm{~nm}$ to $18 \mathrm{~nm}$, whereas the exciton radius $a_{B}=\hbar / \sqrt{2 \mu E_{b}}$ only changes from $6 \mathrm{~nm}$ to $7 \mathrm{~nm}$ in the same well width range (calculated using values of exciton binding energies $E_{b}$ from Ref. 22). We note that analysis based on the separation of the exciton wave function into relative and center-of-mass coordinates might not be valid in our narrowest wells $(<16 \mathrm{ML})$, where the confinement potential exceeds the exciton binding energy.

The simulations of Castella and Wilkins ${ }^{15}$ show that the shape of the exciton spectra is sensitive to the ratio between the correlation length scale $\xi$ of the disordered quantum well potential and the localization length $\xi_{0}$. We note that a doublet structure in the exciton spectra is expected when the $\xi / \xi_{0} \approx 1 / 2$ and a ML splitting occurs when $\xi / \xi_{0} \approx 1$, with the splitting becoming gradually more defined as the ratio increases. By comparing the shape of our measured spectra (Figs. 2 and 6) with the simulations of Ref. 15 we can identify distinct regimes of potential fluctuations, corresponding to different $\xi / \xi_{0}$ ratios. Using localization lengths determined from Eq. (2) we can derive approximate length scales of interface roughness in our quantum wells.

(i) In the continuously grown quantum wells, the interface roughness is fully averaged over the exciton area and no splitting is observed in the PL spectra. By putting $V_{0}$ in Eq. (2) equal to the exciton-averaged potential variation, $\sigma_{E}$, determined from the lineshape fit presented in the previous section, we deduce that $\xi_{0}$ varies from $14 \mathrm{~nm}$ in narrow wells to $30 \mathrm{~nm}$ in wider wells. The degree of localization was also deduced independently via the localization energy parameter $\eta$ in the lineshape fit. The relationship between the localization length and the localization radius is given by $\Delta R$ $=\left(\xi_{0} / \pi\right) \sqrt{\sigma_{E} / \eta}$. We observe a constant $\sigma_{E} / \eta$ ratio for all well widths, yielding a direct proportionality between $\Delta R$ and $\xi_{0}$. From the fact that we observe a single asymmetric ODOS peak, even in our narrowest wells where the localization length is shortest, we conclude, by comparison with simulations, that the typical correlation length in continuously grown wells is $\xi \leq \xi_{0} / 4 \approx 3 \mathrm{~nm}$, much smaller than the localization length.

(ii) Interrupting growth at the bottom interface slightly changes the luminescence characteristics, most notably in wells under $7 \mathrm{~nm}$ thickness. We are therefore moving out of the small island regime, where the exciton in insensitive to the underlying potential fluctuation. Since no evidence of peak splitting is observed, the localization length in the narrow wells can still taken to be around $14 \mathrm{~nm}$. The fact that no splitting is observed furthermore implies that $\xi_{0} / \xi<1 / 2$ so we estimate the correlation length in this case to be $4-6 \mathrm{~nm}$.

(iii) Wells with GI at the top interface show a weak splitting of the exciton peak, but no pinning of peak energies. The shape of the spectra agrees with simulation results in an intermediate regime, where island sizes approach half of the localization length, around $6-8 \mathrm{~nm}$ in this case. Here, the island size is also approaching the exciton diameter and the averaging of the potential becomes less important.

(iv) When growth is interrupted at both interfaces, islands become sufficiently large for the ODOS to split into discrete levels. From Fig. 6 we observe that the ML splitting is more pronounced in thinner wells, where the localization length is shorter. Comparison with simulations indicates that correlation lengths comparable to $\xi_{0}$ in thicker wells and up to $2 \xi_{0}$ in thinner wells match our observations, giving a typical potential correlation length in all wells of $15-20 \mathrm{~nm}$.

Growth-interruption is known to result in the formation of large growth islands on the GaAs surface, with sizes of more than $50 \mathrm{~nm} .^{12,13}$ In samples with GI at both interfaces, we therefore attribute the observed $15-20 \mathrm{~nm}$ correlation length of the quantum well potential to the typical island size on the GI AlAs surface. When growth is not interrupted at the bottom interface (case iii), the island size is reduced to $6-8 \mathrm{~nm}$. During GI, the AlAs surface therefore relaxes by enlarging the island size by a factor of $2-3$, consistent with a slow surface diffusion of Al. Using GI only at the bottom interface (case ii), the observed potential correlation length is $4-6 \mathrm{~nm}$. Knowing that typical island sizes on the GI AlAs surface are significantly larger, this length represents the island size on the non-GI GaAs surface. This is in agreement with STM studies on non-GI GaAs surfaces (grown at $580{ }^{\circ} \mathrm{C}$ ) which have shown typical island sizes of $3-6 \mathrm{~nm} .{ }^{13}$ The deduced roughness length scales of $4-6 \mathrm{~nm}$ and $6-8 \mathrm{~nm}$ at non-GI top and bottom interfaces, respectively, are also consistent with the observed $\leqslant 3-\mathrm{nm}$ combined potential correlation length in Wafer 1.

From the small variations in ML peak positions within each wafer and between wafers grown under similar conditions, we conclude that atomically smooth monolayer-high islands are formed on both AlAs and GaAs surfaces upon GI during MBE growth. A significant degree of nanoroughness on the growth islands would necessarily result in greater 
fluctuations of the ML peak positions. Although atomically smooth AlAs surfaces can be formed during growth, atomicscale nanoroughness still arises during overgrowth because of the segregation of $\mathrm{Al}$ atoms into the GaAs quantum well. This process results in an atomically rough interface, extending through approximately $2 \mathrm{ML}$, according to our simulations. Similarly, segregation of Ga atoms into the AlAs barrier also takes place at the top interface. The segregation length of Ga into AlAs is longer than that of $\mathrm{Al}$ in $\mathrm{GaAs}$, but the effect of Ga segregation on transition energies is smaller since it occurs in the barrier rather than the quantum well. A higher degree of segregation might be responsible for a blueshift of ML peaks observed with increasing growth temperatures. ${ }^{11,18}$ The peak shift reported in these studies, however, is considerably larger than predicted by our simulations. The combination of surface diffusion during GI resulting in large atomically flat growth islands and atomicscale segregation during overgrowth is the origin of the bimodal character of the interface roughness. In the case of non-GI quantum wells, the combined effects of small islands on as-grown surfaces and segregation will result in an nanorough interface of increased thickness and a smoother quantum well potential, consistent with a red-shift of luminescence upon GI, observed by us (Fig. 1) and others, e.g., Ref. 10.

\section{CONCLUSIONS}

From the above discussion we stress the following points, frequently overlooked in the previous literature:

(i) Shifting of ML peaks and observation of sub-ML splitting in growth-interrupted quantum wells is due to the finite quantization energy of excitons localized in monolayer islands with sizes exceeding the exciton localization length. The localization length, which is determined by the monolayer splitting and the exciton mass, can be considerably larger than the exciton diameter. Positions of ML peaks for negligible in-plane quantization energy are reproducible in wafers grown under similar conditions.

(ii) Observation of interface nanoroughness in quantum wells does not imply nanoroughness on free as-grown surfaces. Instead, substantial interface roughness on the atomic scale is unavoidably introduced through surface segregation during growth, resulting in a bimodal distribution of the inplane disorder potential correlation length, especially important in growth-interrupted quantum wells.

(iii) Surface segregation changes the shape of the quantum well potential, increasing ground-state transition energies as compared to a square quantum well potential with the same deposited thickness.

In summary, we have argued that atomically smooth growth islands can be formed on free AlAs and GaAs surfaces following growth interruption. The resulting correlation length of the well potential is sufficiently large, compared to the exciton localization length, to cause a monolayer splitting of the optical density of states. Segregation during the growth of the wells is responsible for formation of nanorough quantum well interfaces. A bimodal interface roughness therefore arises naturally when large atomically smooth islands are formed during growth. Furthermore, the segregation modifies the shape of the potential well, causing a blueshift of transition energies.

\section{ACKNOWLEDGMENTS}

The samples were grown at III-V Nanolab, a joint laboratory between Research Center COM and the Niels Bohr Institute, Copenhagen University. The authors wish to thank Dr. C.B. Sørensen for his assistance with the MBE growth. This work was supported by the Danish Ministries of Industry and Research under the framework of CNAST (Center for Nanostructures).
*Present address: Lehrstuhl für Experimentelle Physik EIIb, Universität Dortmund, Otto-Hahn-Str. 4, 44227 Dortmund, Germany.

${ }^{1}$ A comprehensive review of luminescence studies of heterointerfaces until 1990 can be found in M. A. Herman, D. Bimberg, and J. Christen, J. Appl. Phys. 70, R1 (1991).

${ }^{2}$ R.F. Kopf, E.F. Schubert, T.D. Harris, and R.S. Becker, Appl. Phys. Lett. 58, 631 (1991).

${ }^{3}$ D. Gammon, B. V. Shanabrook, and D. S. Katzer, Appl. Phys. Lett. 57, 2710 (1990).

${ }^{4}$ C.A. Warwick, W.Y. Jan, A. Ourmazd, and T.D. Harris, Appl. Phys. Lett. 56, 2666 (1990).

${ }^{5}$ C.A. Warwick, R.F. Kopf, Appl. Phys. Lett. 60, 386 (1992).

${ }^{6}$ B. Orschel, G. Oelgart, and R. Houdré, Appl. Phys. Lett. 62, 843 (1993).

${ }^{7}$ J.C. Woo, S.J. Rhee, Y.M. Kim, H.S. Ko, W.S. Kim, and D.W. Kim, Appl. Phys. Lett. 66, 338 (1995).

${ }^{8}$ Z.L. Yuan, Z.Y. Xu, B.Z. Zheng, C.P. Luo, J.Z. Xu, W. Ge, P.H. Zhang, X.P. Yang, J. Appl. Phys. 79, 1073 (1996).

${ }^{9}$ K. Fujiwara, H.T. Grahn, and K.H. Ploog, Phys. Rev. B 56, 1081 (1997).

${ }^{10}$ M.K. Chin and C.P. Luo, J. Lumin. 79, 233 (1998).

${ }^{11}$ D. Gammon, B.V. Shanabrook, and D.S. Katzer, Phys. Rev. Lett. 67, 1547 (1991)
${ }^{12}$ D. Bimberg, F. Heinrichsdorff, R.K. Bauer, D. Gerthsen, D. Stenkamp, D.E. Mars, and J.N. Miller, J. Vac. Sci. Technol. B 10, 1793 (1992).

${ }^{13}$ R.F. Kopf, E.F. Schubert, T.D. Harris, R.S. Becker, and G.H. Gilmer, J. Appl. Phys. 74, 6139 (1993).

${ }^{14}$ R. Grousson, V. Voliotis, N. Grandjean, J. Massies, M. Leroux, and C. Deparis, Phys. Rev. B 55, 5253 (1997).

${ }^{15}$ H. Castella and J.W. Wilkins, Phys. Rev. B 58, 16186 (1998).

${ }^{16}$ A. Melliti, M.A. Maaref, and R. Planel, J. Lumin. 78, 25 (1998).

${ }^{17}$ J. Zhang, P. Dawson, J.H. Neave, K.J. Hugill, I. Galbraith, P.N. Fawcett, and B.A. Joyce, J. Appl. Phys. 68, 5595 (1990).

${ }^{18}$ D.S. Katzer, D. Gammon, B.V. Shanabrook, J. Vac. Sci. Technol. B 10, 800 (1992).

${ }^{19}$ A. Manassen, E. Cohen, A. Ron, E. Linder, and L.N. Pfeiffer, Phys. Rev. B 54, 10609 (1996).

${ }^{20}$ R.F. Schnabel, R. Zimmermann, D. Bimberg, H. Nickel, R. Lösch, and W. Schlapp, Phys. Rev. B 46, 9873 (1992).

${ }^{21}$ J.R. Jensen, J.M. Hvam, and W. Langbein, J. Appl. Phys. 86, 2584 (1999).

${ }^{22}$ M. Gurioli, J. Martinez-Pastor, M. Colocci, A. Bosacchi, S. Franchi, and L.C. Andreani, Phys. Rev. B 47, 15755 (1993).

${ }^{23}$ T. Ruf, J. Spitzer, V.F. Sapega, V.I. Belitsky, M. Cardona, and K. Ploog, Phys. Rev. B 50, 1792 (1994). 
${ }^{24}$ K. Brunner, G. Abstreiter, G. Böhm, G. Tránkle, G. Weimann, and Appl. Phys. Lett. 64, 3320 (1994).

${ }^{25}$ H.F. Hess, E. Betzig, T.D. Harris, L.N. Pfeiffer, and K.W. West, Science 264, 1740 (1994).

${ }^{26}$ D. Gammon, E.S. Snow, and D.S. Katzer, Appl. Phys. Lett. 67, 2391 (1995).
${ }^{27}$ U. Jahn, S.H. Kwok, M. Ramsteiner, R. Hey, H.T. Grahn, and E. Runge, Phys. Rev. B 54, 2733 (1996).

${ }^{28}$ R. Zimmermann and E. Runge, J. Lumin. 60, 320 (1994).

${ }^{29}$ Al.L. Efros, C. Wetzel, and J.M. Worlock, Phys. Rev. B 52, 8384 (1995). 